\title{
openheart MRI-guided pulmonary vein isolation for atrial fibrillation: what is good enough? An early health technology assessment
}

\author{
Steven Wenker (D , ${ }^{1}$ Chris van Lieshout, ${ }^{2}$ Geert Frederix, ${ }^{2}$ Jeroen van der Heijden, ${ }^{1}$ \\ Peter Loh, ${ }^{1}$ Steven A J Chamuleau, ${ }^{1}$ Frebus van Slochteren ${ }^{1}$
}

To cite: Wenker S, van Lieshout C, Frederix G, et al. MRI-guided pulmonary vein isolation for atrial fibrillation: what is good enough? An early health technology assessment. Open Heart 2019;6:e001014. doi:10.1136/

openhrt-2019-001014

Received 28 January 2019 Revised 29 May 2019 Accepted 12 September 2019

Check for updates

(C) Author(s) (or their employer(s)) 2019. Re-use permitted under CC BY-NC. No commercial re-use. See rights and permissions. Published by BMJ.

${ }^{1}$ Department of Cardiology, University Medical Center Utrecht, Utrecht, The

Netherlands

2 Julius Centre, University Medical Center Utrecht, Utrecht, The Netherlands

Correspondence to Dr Steven Wenker; s.wenker@ umcutrecht.n

\section{ABSTRACT}

Next to anticoagulation, pulmonary vein isolation (PVI) is the most important interventional procedure in the treatment of atrial fibrillation (AF). Despite widespread clinical application of this therapy, patients often require multiple procedures to reach clinical success. In contrast to conventional imaging modalities, MRI allows direct visualisation of the ablation lesion. Therefore, the use of real-time MRI to guide cardiac electrophysiology procedures may increase clinical effectiveness. An essential aspect, from a decision-making point of view, is the effect on costs and the potential cost-effectiveness of new technologies. Generally, health technology assessment (HTA) studies are performed when innovations are close to clinical application. However, early stage HTA can inform users, researchers and funders about the ultimate clinical and economic potential of a future innovation. Ultimately, this can guide funding allocation. In this study, we performed an early HTA evaluate MRIguided PVIs.

Methods We performed an economic evaluation using a decision tree with a time-horizon of 1 year. We calculated the clinical effectiveness (defined as the proportion of patients that is long-term free of $\mathrm{AF}$ after a single procedure) required for MRI-guided PVI to be cost-effective compared with conventional treatment.

Results Depending on the cost-effectiveness threshold (willingness to pay for one additional quality-of-life adjusted life year (QALY), interventional MRI (iMRI) guidance for PVI can be cost-effective if clinical effectiveness is $69.8 \%$ (at $€ 80000 / Q A L Y$ ) and $77.1 \%$ (at $€ 20000 /$ QLY), compared with 64\% for fluoroscopyguided procedures.

Conclusion Using an early HTA, we established a clinical effectiveness threshold for interventional MRI-guided PVIs that can inform a clinical implementation strategy. If crucial technologies are developed, it seems plausible that iMRI-guided PVls will be able to reach this threshold.

\section{INTRODUCTION}

Atrial fibrillation (AF) is the most common cardiac arrhythmia. It is characterised by rapid and disorganised electrical activity in the cardiac atria. AF can lead to thrombus

\section{Key questions}

What is already known about this subject?

- Interventional MRI has been investigated since the early 2000s. Currently, it is technically feasible to guide several commonly performed procedures using MRI (eg, flutter ablations or cardiac biopsy). However, not much is known about costeffectiveness. As direct procedural costs are likely higher for MRI-guided procedures, in order to be cost-effective, outcomes would likely need to be better than using current techniques (which could lead to better quality of life and to reduced subsequent healthcare expenditures).

What does this study add?

- Using a decision tree model, we analysed potentia cost-effectiveness of MRI guidance of pulmonary vein isolations for atrial fibrillation. The required clinical benefit of the procedure in order to reach costeffectiveness depends on the willingness to pay for one additional quality-of-life adjusted life year. We make estimates for required clinical effectiveness based on realistic bounds of the willingness to pay.

How might this impact on clinical practice?

- Clinical translation of (technical) innovations only make sense if researchers and commercial partners believe it is potentially cost-effective. Analysing this question early in development of new technology allows time, effort and costs to be saved if new technologies are unlikely to be successfully implemented.

formation in the atria (specifically in the left atrial appendage) and may cause cerebral infarction as well as heart failure. AF and its sequelae are a significant cause of significant mortality and morbidity. The European Heart Network estimates that over 33.5 million people live with AF worldwide. ${ }^{1}$

Two main treatment strategies for AF exist currently: rate control and rhythm control. In rate control, medication is used to control conduction to the ventricles and decrease the 
ventricular heart rate while AF persists. In rhythm control medication, cardioversion, catheter ablation and sometimes surgery are used to terminate $\mathrm{AF}$ and to maintain sinus rhythm. ${ }^{2}$

The most common interventional treatment of $\mathrm{AF}$ is catheter ablation of the pulmonary vein ostia. In this so-called pulmonary vein isolation (PVI) procedure, the four pulmonary veins are electrically isolated from the rest of the atrial myocardium. The pulmonary veins often contain triggers that initiate and sustain the atrial arrhythmia. In about $87.5 \%$ of patients, PVI is a successful strategy for reducing the overall burden of $\mathrm{AF}^{3}$ However, a significant portion of these patients need more than one procedure. In $90 \%$ of redo procedures, a pulmonary vein reconnection can be identified. ${ }^{34}$ In a pulmonary vein reconnection, a pulmonary vein that was electrically isolated after the prior ablation procedure can regain its connection to the rest of the atrium because not all the acutely ablated area turns into permanent scar. Although the causal relationship is still topic of discussion, ${ }^{5}$ it is generally accepted that pulmonary vein reconnection is a major contributor to recurrence of AF.

Cardiac interventions generally use fluoroscopy for treatment guidance. During ablation procedures, fluoroscopy is often supplemented with electroanatomical mapping (EAM). EAM allows the measurement and visualisation of local electrical activity. Treatment guidance by MRI (interventional MRI (iMRI)) is an alternative for fluoroscopy and has several distinct advantages when compared with fluoroscopy: it can provide anatomical information in three-dimensional (3D), it has excellent soft tissue visualisation and it does not use harmful ionising radiation. iMRI can be combined with EAM. Interventional MRI is already clinically being applied for a multitude of right-sided cardiac interventions. ${ }^{6}$ In cardiac ablations, it offers a unique potential: the possibility to visualise the tissue characteristics prior to the ablation and the formation of the ablation lesion formation in real-time. ${ }^{7}$ We hypothesise that, if iMRIEAM-guided PVI therapy allows direct assessment of the radiofrequency (RF) ablation lesion, effectiveness of RF ablation could be improved compared with fluoroscopyEAM-guided procedures.

Cost-effectiveness has become a central question for new innovations due to rapidly increasing healthcare costs. Cost effects of new innovations (such as iMRI-EAM guided PVI therapy) should be balanced with clinical effects. Due to resource constraints, willingness to pay for one additional quality-adjusted life year (QALY) cannot be taken for granted. Health technology Assessments (HTAs) are a tool to evaluate the additional cost or cost savings and to compare them to clinical benefits. As such they are very often used by decision makers and are endorsed by the WHO. ${ }^{89}$ Generally, such HTAs are performed for technologies or innovations that are close to clinical translation or after one or more clinical trials have been completed. At that stage, a clear picture can be acquired of financial and health benefits and costs. However, if an innovation turns out to be not costeffective, many years of research may have already been invested.

In contrast to the 'late stage' use, HTAs can also be used at an 'early stage', when both costs and effects of the innovation are still largely unknown (early HTA). These early HTAs aim to evaluate potential costs and benefits of technologies that are not yet (fully) developed. This information can be used to effectively allocate research budget to innovations that have high potential and to guide further development past potential bottlenecks. Main outcome of HTAs generally is the incremental cost-effectiveness ratio (ICER), in which costs and health effects of current standard of care are compared with the new intervention. The ICER calculates the cost per QALY and therefore also indicates the total amount of effect to be gained by an innovation.

In this report, we performed an early HTA and investigated at which clinical effectiveness (ie, at what recurrence rate) iMRI-EAM guidance becomes cost-effective when compared with fluoroscopy-EAM-guided PVI interventions. We defined the clinical effectiveness as the percentage of patients free of $\mathrm{AF}$ after the first procedure.

\section{METHODS}

Various HTA methods can be used to link both costs and effects. In this instance, a standard HTA decision tree was constructed in Microsoft Excel (Microsoft, Redmond, Washington, USA). A decision tree is a form of analytical model, in which distinct branches are used to represent a potential set of outcomes for a patient or patient cohort. A decision tree consists of a series of nodes where branches meet: each node may take the form of a choice (a decision about which alternative intervention to use) or a probability (an event occurring or not occurring, governed by chance). Costs and outcomes are assigned to each segment of each branch, including the end ('leaf') of each branch.

The model consisted of two main branches representing the fluoroscopy-EAM-guided therapy and the iMRI- EAM therapy (figure 1). In both branches, all the possible subsequent outcomes of the therapeutic steps were modelled. Patients entered the model in one of the two main branches. After the initial procedure, the procedural complications and outcomes were modelled. Patients who did not benefit from the initial procedure either underwent a second procedure after 6 months or pharmaceutical treatment of $\mathrm{AF}$ was given according to current guidelines. Complications were modelled as a weighted average of: vascular complications, cardiac tamponade, cerebral embolism and pulmonary vein stenosis.

The primary outcome of the model was the minimal required 'clinical effectiveness' of MRI-guided PVI to reach a predefined cost-effectiveness threshold (CET). The clinical effectiveness is expressed as the proportion of patients being free of $\mathrm{AF}$ after the first procedure (ie, 

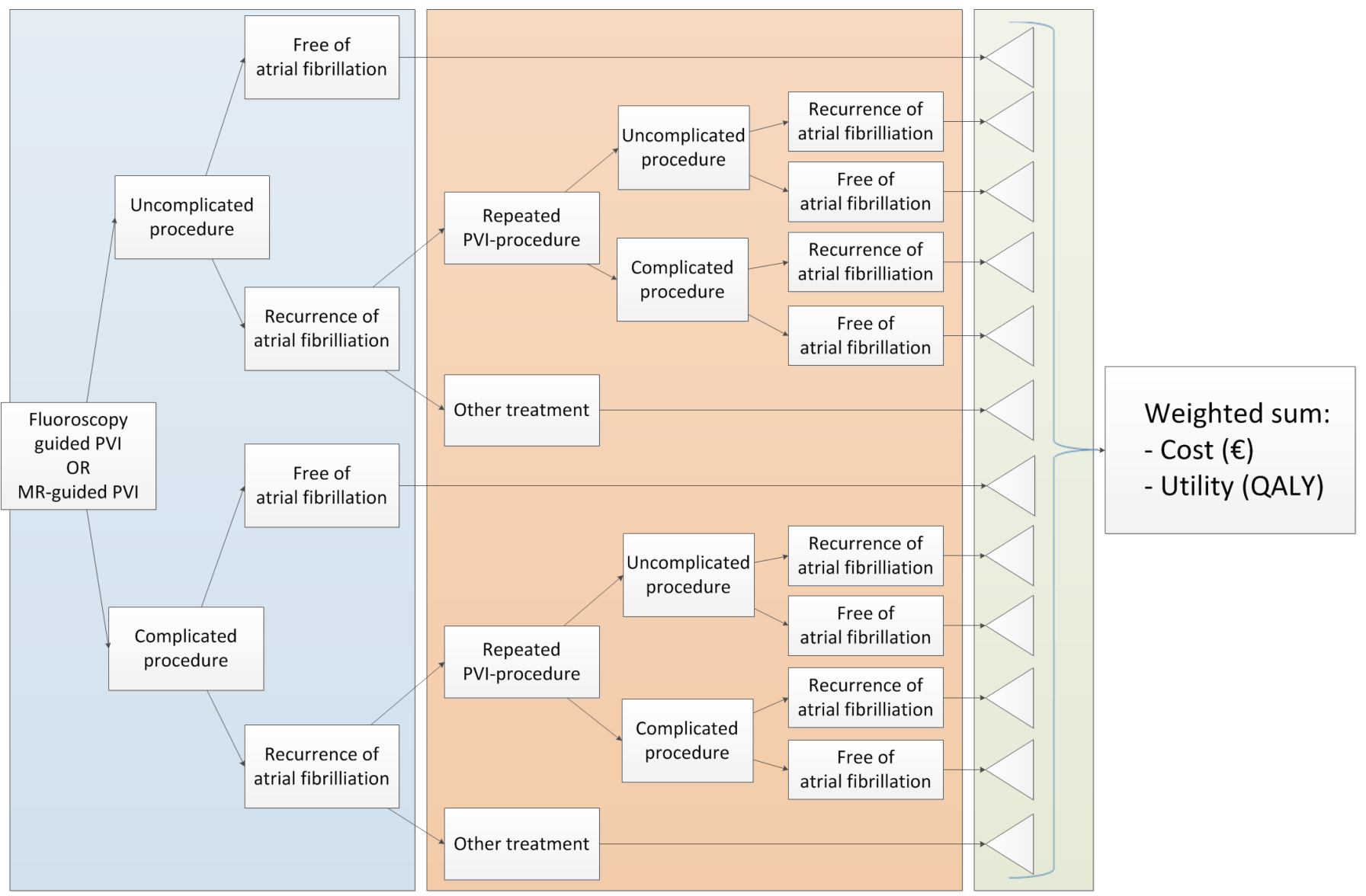

Figure 1 Decision tree model. Fluoroscopy-guided treatment and MR-guided treatment both had an identical main branch. $\mathrm{PVI}$, pulmonary vein isolation; QALY, quality-adjusted life year.

one minus the recurrence rate). Within the iMRI branch of the decision, the clinical effectiveness is allowed to vary. Note that, as this parameter increases, cost goes down, utility increases and therefore cost per QALY decreases.

To compare cost-effectiveness for new innovations, the ICER can be calculated. The ICER represents the additional costs incurred for each QALY gained. It is calculated by dividing the difference in cost between the old and new pathway by the difference in health outcomes (ie, the difference in utility, measured in QALY (see equation 1)).

Equation 1. calculation of the ICER

$$
I C E R=\frac{\text { Total Costs } s_{i M R I}-\text { Total Costs } \text { Conventional }}{\text { Total EffectsinRI }- \text { Total Effect Conventional }}=\frac{\Delta \text { Costs }}{\Delta \text { Effects }}
$$

In our analysis, the ICER was set to equal a threshold varying between 20000 to $80000 € /$ QALY, and the model was used to find the value for the "clinical effectiveness' that satisfies this equation. In other words, we used the model to find the 'clinical effectiveness' at which health gains achieved were less expensive than the different thresholds. These thresholds are based on recommendations for reimbursement by the Dutch Council for public health and healthcare (Raad voor de Volksgezondheid en Zorg).

\section{MODEL INPUTS}

All data used to determine node probabilities (table 1) correspond to earlier research conducted at University Medical Center (UMC) Utrecht. ${ }^{34}$ The UMC Utrecht is a large tertiary, academic medical centre in the Netherlands. Over this period (2010-2014), around 110 primary PVI procedures were performed yearly. Rates of recurrence and complications are comparable with rates mentioned elsewhere in the literature. ${ }^{10}$

\section{COST ESTIMATES}

The costs for the treatments and subsequent care were gathered from multiple sources and were based on

\begin{tabular}{ll}
\hline Table 1 Probability inputs & \\
\hline Input parameter & Probabilities \\
\hline $\begin{array}{l}\text { AF free after ablation } \\
\text { (fluoroscopy guided) }\end{array}$ & 0.64 \\
$\begin{array}{l}\text { Complication (weighted } \\
\text { sum) }\end{array}$ & 0.071 \\
$\begin{array}{l}\text { Repeat procedure (after } \\
\text { recurrence) }\end{array}$ & 0.18 \\
\hline
\end{tabular}

Inputs are reused for every procedure.

$\mathrm{AF}$, atrial fibrillation. 


\begin{tabular}{ll}
\hline Table 2 & Cost input parameters \\
\hline Input parameters & Cost \\
\hline Direct procedural costs (conventional treatment) & $€ 9643$ \\
MRI use cost & $€ 1800$ \\
\hline Diagnostics & $€ 612$ \\
Ward stay & $€ 657$ \\
Other treatment & $€ 325$ \\
Complication costs (weighted average) & $€ 626.87$ \\
\hline Vascular & $€ 125$ \\
\hline Tamponade & $€ 2692$ \\
\hline Cerebovascular accident(CVA)/Transient Ischemic & $€ 5816$ \\
Attack (TIA) & $€ 376.43$ \\
\hline Pulmonary vein (PV) stenosis & $€ 0$ \\
\hline Mortality & $€ 667$ \\
\hline Follow-up & $€ 1.86 /$ day \\
\hline Prescription treatment
\end{tabular}

CVA, cerebrovascular accident; TIA, transient ischaemic attacks.

treatment guidelines. Costs of the different processes were determined according to the Zorginstituut Nederland cost manual, ${ }^{11}$ the Dutch online database for medication costs, ${ }^{12}$ and the maximum tariffs set by the Dutch Healthcare Authority (Nederlandse Zorgautoriteit). ${ }^{13}$ All costs were calculated in euros (corrected for inflation to 2017). Specific procedure related costs are based on an internal business case. An overview of all costs can be found in table 2 .

The costs of the iMRI procedure were modelled by taking the control procedure cost, subtracting costs specific to use of the fluoroscopy lab and adding costs specific to the MR-guided procedure. Costs for using the MR machine were calculated from internal cost figures for diagnostic cardiac magnetic resonance, adjusted for 4.5 hours of procedural time. These costs include use of the machine and operator and physician wages. Catheter costs were set to be equal between the procedures.

\section{UTILITY SCORES AND QUALITY OF LIFE}

Utility estimates are indispensable when calculating QALYs. To determine the exact QALY value, the utility value associated with a given health state is multiplied by the years lived in that state. Utilities for each different outcome and events were derived from utilities previously published research. The utilities for AF and sinus rhythm were derived from other ablation cost-effectiveness studies. ${ }^{14}$ The decrement multiplier for cerebrovascular accidents (CVAs) and transient ischaemic attacks (TIA) were taken from a meta-analysis of quality of life estimates in CVA/TIA. ${ }^{15}$ The utility input parameters are summarised in table 3 .
Table 3 Utility inputs for disease states

\begin{tabular}{lcc}
\hline Health state & Utilities & Source \\
\hline AF free & 0.81 & 14 \\
AF recurrence & 0.73 & 14 \\
Other treatment & 0.73 & 14 \\
Complications (weighted sum) & -0.1 & 14,15 \\
\hline
\end{tabular}

\section{COMPLICATIONS}

Complication rates are low during these procedures. Based on expert opinion (PL), we did not expect complications to differ between the fluoroscopy-guided and iMRI-guided procedures. A weighted sum for the costs and the disutility's for all complications was created, and it was treated as a dichotomous variable.

\section{SENSITIVITY ANALYSIS}

To determine which of the input parameters had the largest impact on the cost-effectiveness of MRI guided ablation, one-way sensitivity analyses were performed. Analyses were performed that showed the effect of changing the mean of all individual model input parameters with plus or minus $20 \%$.

\section{RESULTS}

After filling in all parameters, the model showed that total healthcare costs for fluoroscopy-guided ablation was $€ 12773$ with an average utility of 0.776 QALY. For the interventional MRI arm of the model, all cost estimates and all probabilities, except for the recurrence rate (ie, the inverse of clinical effectiveness), were entered. After fixing the CET at a given threshold, we calculated the minimum clinical effectiveness to achieve an ICER below that threshold (so that the costs per QALY gained is less than the threshold) (see eq 1).

With a CET of $€ 20000 /$ QALY, the minimal required success probability for MRI-guided ablation was 0.771 , at a CET of $€ 50000 /$ QALY, this was 0.721 and at $€ 80000 /$ QALY, the required success probability was 0.698 . For each of the different CET thresholds, the corresponding healthcare costs and health gains realised for MRI-guided ablation can be found in table 4 . These costs include all costs incurred in the period modelled (including, eg, probability weighted costs for repeated procedures and follow-up).

\section{Sensitivity analysis}

A probabilistic sensitivity analysis with a Monte Carlo Simulation having 10000 iterations was carried out, but since most parameters do not differ between treatment strategies, little to no variation in ICERs was found.

\section{DISCUSSION}

We performed an early HTA to establish an estimate for the clinical effectiveness required to be cost-effective for 
Table 4 Estimated clinical effectiveness required for costeffectiveness at different cost-effectiveness thresholds

\begin{tabular}{llll}
\hline $\begin{array}{l}\text { Cost- } \\
\text { effectiveness } \\
\text { threshold }\end{array}$ & $\begin{array}{l}\text { Minimal } \\
\text { required } \\
\text { success } \\
\text { probability }\end{array}$ & Total costs & $\begin{array}{l}\text { Utility } \\
\text { (QALY) }\end{array}$ \\
\hline$€ 20000 /$ QLYY & 0.771 & $€ 12983$ & 0.786 \\
$€ 50000 /$ ALY & 0.721 & $€ 13102$ & 0.782 \\
$€ 80000 /$ QLYY & 0.698 & $€ 13156$ & 0.780 \\
\hline
\end{tabular}

QALY, quality-adjusted life year.

iMRI guidance of PVI. Since our analysis is performed early in the development of this technique, it is not based on clinical data, as such data are not available. Therefore, our analysis cannot address the question if MRI-guided PVIs are cost-effective directly, rather it is meant to inform researchers in this field about the clinical effectiveness that is required to be cost-effective. Only when researchers believe they can reach this threshold does it make sense to attempt clinical translation.

MRI is considered the gold standard for infarct assessment, and as a diagnostic tool, it is widely used within the field of cardiology. Increasingly, attempts have been made to use MRI for the guidance of invasive procedures. ${ }^{6}$ MRI has distinct advantages when compared to the current standard in treatment guidance: fluoroscopy; MRI offers excellent 3D soft tissue imaging without using harmful ionising radiation. In animal experiments, MRI has been shown to be able to predict histological lesion size and even identify gaps in ablation lines. ${ }^{16}$ If MRI is able to assess lesion quality in real-time, or can help predict lesion durability, for example, using thermography, ${ }^{17}$ it seems plausible that MR guidance can improve effectiveness of catheter ablation.

Recently, more focus is put on the valorisation of research findings. Increasingly, HTAs are a prerequisite for funding or a mandatory part of newly funded projects. ${ }^{18}$ Here, we analysed the use of MR guidance and its cost-effectiveness for the use of PVIs in case of AF. Our analysis was focused on this procedure because it is a high-volume procedure performed in many centres globally, but approximately $30 \%$ of patients end up requiring one or multiple repeated procedures to achieve clinical success. ${ }^{34}$ Any innovation that can reduce this number can have major impact on quality of life for patients as well as healthcare costs and should be pursued. Many technical solutions have been proposed and investigated recently such as contact-forcing catheters, 3D navigational systems and other forms of lesion formation (such as cryoablation).

To date, no reports have evaluated the use of iMRI for ablation of AF, but several groups have reported on ablation of atrial flutter using iMRI. ${ }^{19-21}$ Although earlier works reported significantly longer procedural times than fluoroscopy-guided treatment, ${ }^{20} 21$ the most recent case series $^{19}$ shows procedural times that are comparable with fluoroscopy-guided treatment (45.9 min vs $48.4 \mathrm{~min}$ for iMRI-guided and conventional treatment, respectively) with similar results with regards to direct procedural success and short-term follow-up. In this publication, a strong learning effect is noted, and procedural times initially decreased sharply and plateaued after 12 procedures. Although a PVI is a more complicated procedure, these data suggest that treatment times may be comparable between iMRI-guided treatment and conventional treatment after a relatively short run-in period.

We hypothesise that there are two ways in which PVI might fail to relieve AF. The first way is when incomplete lesion sets lead to insufficient electrical isolation between the pulmonary veins and the rest of the left atrium. The second way is when other drivers of $\mathrm{AF}$ are (also) present in a significant portion of patients and even complete isolation of the pulmonary veins is not sufficient to treat all patients satisfactorily. Under this hypothesis, we expect that most improvements in iMRI-guided procedures would be due to more complete lesion sets in the primary procedure and that a smaller proportion of patients would require repeated procedures but that the cumulative effect after repeated procedures remains similar.

The difference between treatment options then arises at discrete and predictable timepoints that are well modelled by a decision tree model. Other model types (eg, Markov chain model) can be used to model exposure to continuous risks over time. In this analysis, we did not assume any time-dependent risks. Under our assumptions, most of the benefit would be captured in a relatively short time period, but the nature of possible future benefits is by definition speculative. It is plausible that a longer time horizon under different assumptions would yield different results.

Although a non-negligible proportion of patient undergoes more than one repeated procedure, these number decrease exponentially and therefore have decreasing impact on this type of analyses. To account for multiple repeated procedures would require assumptions about the efficacy of repeated iMRI-guided procedures, which again would be difficult to substantiate.

Outcomes and complication rates of ablations may vary between institutions. The data used in our model were gathered from a publication reporting on outcomes in our own institutions and are generally in line with other reports. ${ }^{102223}$

In the Netherlands, the CET for reimbursement varies according to disease severity between $€ 20000$ and $€ 80000$ per quality of life adjusted life year. ${ }^{11} 1324$ In this perspective, at a utility of 0.71 , AF is a relatively mild disease. Therefore, we expect the CET in practice to be near the lower end of this range. Our analysis shows that, at the lower end of this range (€20 000 per QALY), iMRIguided procedures need to achieve long-term success after the first procedure in $77.1 \%$ of patients to be costeffective. For reference, the number used in our model for fluoroscopy guided procedures is $64 \% .^{3}$ 
It seems unlikely that these thresholds can be reached with currently available technologies as essential parts of the required tools for PVI are currently unavailable. Over the past decade, the first applications of interventional MRI are getting close to clinical translation (atrial flutter ablations ${ }^{19}$ ) or have already been successfully adopted in select medical centres (diagnostic right sided heart catheterisation). ${ }^{25}$ However, these applications are currently limited to relatively low complexity, right-sided procedures. In contrast, for ablations for $\mathrm{AF}$, access to the left atrium is required, and a more specialised toolkit is necessary. A trans-septal puncture kit, a circular mapping catheter and a pressuresensitive, irrigated radiofrequency ablation catheter are not available for use in the MRI and would need to be developed to be able to compete with fluoroscopy. Lesion mapping techniques, especially for the thinwalled atria, would need to be further researched and validated to make optimal use of the potential of MRI for treatment guidance.

Our analysis is not meant to determine if iMRI-guided procedures are cost-effective today; instead, we intend to set the bar researchers and developers within the field of iMRI should strive to reach.

\section{Limitations}

In our analysis, we used a time horizon of 1 year. If we suppose that MRI guidance reduces recurrence mainly by recognition of insufficient lesions during the initial procedure, we expect most of the benefit of MRI guidance to be expressed in a reduced need for repeated procedures. However, this does not account for other methods by which MRI guidance may improve outcomes, for example, by identification of additional substrate. Various highly relevant factors are also unaccounted for in this model, such as ergonomics or radiation exposure (both for patient and operator). This analysis applies first and foremost to our own institution as specific parts of the costs (such catheter prices) may differ between institutions.

Upfront costs (eg, for acquisition of an MR scanner or catheterisation lab) were not analysed. These costs were excluded because acquisition costs may vary wildly between countries and institutions and depend heavily on the type of room and equipment acquired (eg, hybrid MRI/fluoroscopy of MRI only). Furthermore, such analysis would require assumptions about how the machine is used in practice (ie, how many cardiac and non-cardiac procedures are performed, how often it is used for scanning and so on). Instead, this analysis supposes a hospital that has both treatment modalities available and costs of acquisition are accounted for in use cost of both modalities, basing the cost-estimates on local experience. Although an interventional MRI suite is presumably not available in many general hospitals yet, several groups from various countries have published case series from cardiac procedures in interventional MRI suites. ${ }^{19} 2025$

\section{CONCLUSIONS}

Our early HTA shows that the use of interventional MRI to guide PVI for treatment of AF is likely to be cost-effective if clinical effectiveness of MRI-guided procedures is above $72.1 \%$ (depending on CET, range: $69.8 \%-77 . \%$ ). Given the current effectiveness of fluoroscopy-guided procedures of $64 \%$, if MRI can predict gaps in ablation lesions, this appears to be a realistic goal.

Contributors SW: conceptualisation, planning, data acquisition, analysis, writing and submission. CvL: conceptualisation, planning, data acquisition and analysis. GF: conceptualisation and technical supervision. PL: data acquisition, providing medical background and manuscript revision. JvdH: providing medical background and manuscript revision. SAJC and FvS: conceptualisation, planning, project supervision and manuscript revision.

Funding This work was conducted within the Topconsortia for Knowledge and Innovation's - Life Sciences \& Health (TKI LSH) framework and with financial support of the National Health Service, Interuniversity Cardiology Institute of the Netherlands (ICIN) - Netherlands Heart Foundation and partners of the Mrl Guided RegenerAtive ThErapy (MIGRATE) consortium.

Competing interests None declared.

Patient consent for publication Not required.

Provenance and peer review Not commissioned; externally peer reviewed.

Data availability statement Data are available on reasonable request.

Open access This is an open access article distributed in accordance with the Creative Commons Attribution Non Commercial (CC BY-NC 4.0) license, which permits others to distribute, remix, adapt, build upon this work non-commercially, and license their derivative works on different terms, provided the original work is properly cited, appropriate credit is given, any changes made indicated, and the use is non-commercial. See: http://creativecommons.org/licenses/by-nc/4.0/.

ORCID iD

Steven Wenker http://orcid.org/0000-0003-4671-6322

\section{REFERENCES}

1 Chugh SS, Havmoeller R, Narayanan K, et al. Worldwide epidemiology of atrial fibrillation: a global burden of disease 2010 study. Circulation 2014;129:837-47.

2 Kirchhof P, Benussi S, Kotecha D, et al. 2016 ESC guidelines for the management of atrial fibrillation developed in collaboration with EACTS. Eur Heart J 2016;37:2893-962.

3 Teunissen C, Kassenberg W, van der Heijden JF, et al. Five-Year efficacy of pulmonary vein antrum isolation as a primary ablation strategy for atrial fibrillation: a single-centre cohort study. Europace 2016;18:1335-42.

4 Teunissen C, Clappers N, Hassink RJ, et al. A decade of atrial fibrillation ablation shifts in patient characteristics and procedural outcomes. Netherlands Hear J 2017;25:559-66.

5 Nery PB, Belliveau D, Nair GM, et al. Relationship Between Pulmonary Vein Reconnection and Atrial Fibrillation Recurrence: A Systematic Review and Meta-Analysis. JACC Clin Electrophysiol 2016;2:474-83.

6 Rogers T, Lederman RJ. Interventional CMR: clinical applications and future directions. Curr Cardiol Rep 2015;17.

7 Vergara GR, Vijayakumar S, Kholmovski EG, et al. Real-Time magnetic resonance imaging-guided radiofrequency atrial ablation and visualization of lesion formation at 3 tesla. Heart Rhythm 2011;8:295-303.

8 WHO. Health technology assessment: HTa definitions. Available: http://www.who.int/health-technology-assessment/about/Defining/ en/

9 SIXTY-SEVENTH World health assembly WHA; 24 may 2014; agenda item 15.7; health intervention and Technology assessment in support of universal health coverage.

10 Calkins H, Hindricks G, Cappato R, et al. 2017 HRS/EHRA/ECAS/ APHRS/SOLAECE expert consensus statement on catheter and surgical ablation of atrial fibrillation. Heart Rhythm 2017;14:e275-444 https://doi.org/

11 Hakkaart-van Roijen L, van der Linden N, Bouwmans C, et al. Kostenhandleiding: Methodologie van kostenonderzoek 
en referentieprijzen voor economische evaluaties in de gezondheidszorg, 2015.

12 Zorginstituut Nederland. Medicijnkosten.nl. Available: www. medicijnkosten.nl

13 Nederlandse Zorgautoriteit. Tarieventabel dbz-zorgproducten, 2017. Available: https://puc.overheid.nl/nza/doc/PUC_13274_22/1/

14 Reynolds MR, Lamotte M, Todd D, et al. Cost-Effectiveness of cryoballoon ablation for the management of paroxysmal atrial fibrillation. EP Europace 2014;16) :652-9.

15 Tengs TO, Lin TH. A meta-analysis of quality-of-life estimates for stroke. Pharmacoeconomics 2003;21:191-200.

16 Ranjan R, Kholmovski EG, Blauer J, et al. Identification and acute targeting of gaps in atrial ablation lesion sets using a real-time magnetic resonance imaging system. Circulation 2012;5:1130-5.

17 Toupin S, Bour P, Lepetit-Coiffé M, et al. Feasibility of real-time $\mathrm{Mr}$ thermal dose mapping for predicting radiofrequency ablation outcome in the myocardium in vivo. J Cardiovasc Magn Reson 2017;19:1-12.

18 Hartstichting, Brochure. Set up impulse grant, 2018. Available: https://www.hartstichting.nl/getmedia/6b280637-1026-4a54-818bbc396af3a3cf/wetenschappers-cvon-brochure-impulse-grant-2018. pdf

19 Paetsch let al. Clinical workflow and applicability of electrophysiological cardiovascular magnetic resonance-guided radiofrequency ablation of Isthmus-Dependent atrial flutter. European Heart Journal - Cardiovascular Imaging 2018;49:1-10.

20 Chubb H, Harrison JL, Weiss S, et al. Development, Preclinical Validation, and Clinical Translation of a Cardiac Magnetic Resonance - Electrophysiology System With Active Catheter Tracking for Ablation of Cardiac Arrhythmia. JACC Clin Electrophysiol 2017;3:89-103.

21 Hilbert S, Sommer P, Gutberlet M, et al. Real-Time magnetic resonance-guided ablation of typical right atrial flutter using a combination of active catheter tracking and passive catheter visualization in man: initial results from a consecutive patient series. Europace 2016:18:572-7.

22 Jiang H, Lu Z, Lei H, et al. Predictors of early recurrence and delayed cure after segmental pulmonary vein isolation for paroxysmal atrial fibrillation without structural heart disease. J Interv Card Electrophysiol 2006;15:157-63.

23 Richter B, Gwechenberger M, Socas A, et al. Frequency of recurrence of atrial fibrillation within 48 hours after ablation and its impact on long-term outcome. Am J Cardiol 2008;101:843-7.

24 Nederlandse Zorgautoriteit. Rapport kosteneffectiviteit in de praktijk. Zorginstituut Nederland, 2015

25 Ratnayaka K, Kanter JP, Faranesh AZ, et al. Radiation-free CMR diagnostic heart catheterization in children. Journal of Cardiovascular Magnetic Resonance 2017;19:1-10. 